\title{
Evaluation of a Self-organized Traffic Light Policy
}

\author{
Michelle Borm \\ Technical University \\ Eindhoven \\ Brendan Patch \\ The University of Queensland \\ University of Amsterdam \\ m.a.m.w.borm@student.tue.nl b.patch@uq.edu.au \\ Ivo Adan \\ Technical University \\ Eindhoven \\ i.j.b.f.adan@tue.nl
}

\begin{abstract}
This paper presents a preliminary assessment of the potential performance gains of a self-organized traffic light policy developed by Lämmer and Helbing. A large amount of data was obtained for a complex real-world intersection that serves as an ideal test-bed for comparison of traffic control policies.
\end{abstract}

We provide evidence that a change in policy may drastically decrease average queue lengths and waiting times, suggesting the self-organized policy is a promising approach to the control of traffic intersections deserving further investigation and potentially implementation.

\section{Categories and Subject Descriptors}

I.6.3 [Simulation and Modelling, Applications]; I.6.8 [Simulation and Modelling, Types of Simulation] Discrete Event; G.3 [Probability and Statistics] Queueing theory

\section{Keywords}

Local control, dynamic control, traffic light policy, traffic network

\section{INTRODUCTION}

Recent increases in computational power and new technologies (e.g. sensors) enable sophisticated traffic light control methodologies. Classically, traffic light policies use periodic schedules based on the assumption that the average number of cars flowing through a road approximates the actual flow at any particular time. This assumption may result in suboptimal traffic light policies. Recent research has focused on relaxing this assumption to develop traffic light policies that depend on real time traffic patterns (see e.g. [1] and the references therein).
In this work we use data from a real-world system to investigate the effectiveness of a self-organized traffic light control policy developed by Lämmer and Helbing [2], which we refer to as "Lämmer's policy". The policy uses the number of approaching cars from each direction of traffic flow to an intersection and the number of waiting cars to prioritize directions and determine the duration of green time per direction for each cycle. The policy prioritizes minimization of total waiting times over the reduction of the total number of cars in queue in all traffic directions. We provide evidence that introducing Lämmer's policy to a network of intersections (Figure 1) in Brisbane (Australia) may reduce congestion. Currently this intersection exhibits congestion at peak times, and is controlled by the Sydney Coordinated Adaptive Traffic System (SCATS) [3].

The exact specification of SCATS is unknown to the authors; we will instead use a SCATS-like policy based on observation in our case study, which is a fixed green times policy.

\section{CASE STUDY: TOOWONG}

We model the traffic intersection depicted in Figure 1 as an open queueing network by viewing cars as customers and the set of traffic lights at intersections as servers. This model is studied computationally through the use of discrete event simulation. For each traffic direction cars are served first-

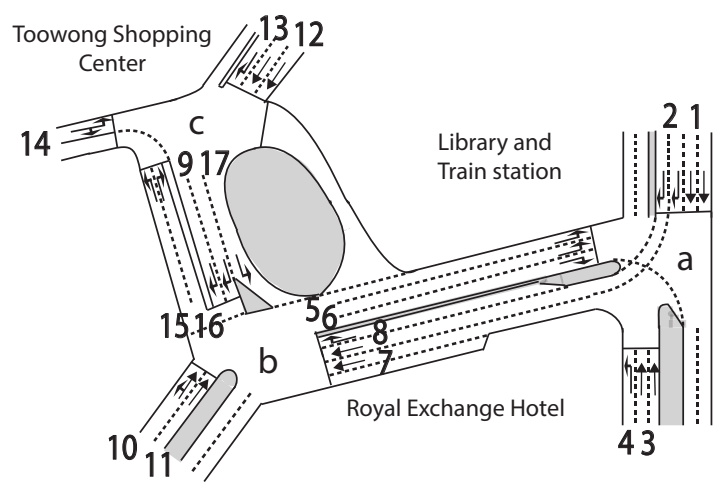

Figure 1: Schematic of our case-study traffic network, the Toowong Shopping Center intersection.

come-first-served and the intersection can only serve predefined subsets of the approaching roads at any given time. We take the topology of the intersection to be fixed. Arrivals are 


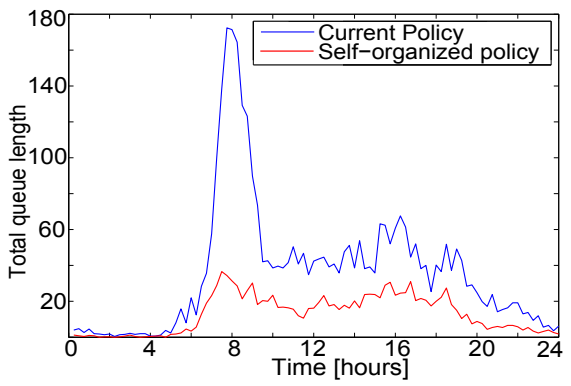

(a) Expected total queue length for the SCATS-like and self-organized policy.

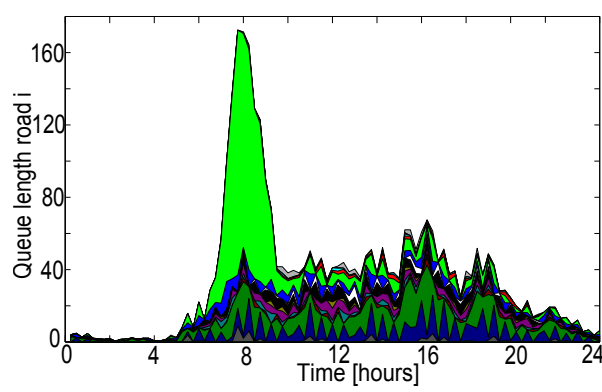

(b) Contribution of each road to the total queue length for the SCATS-like policy.

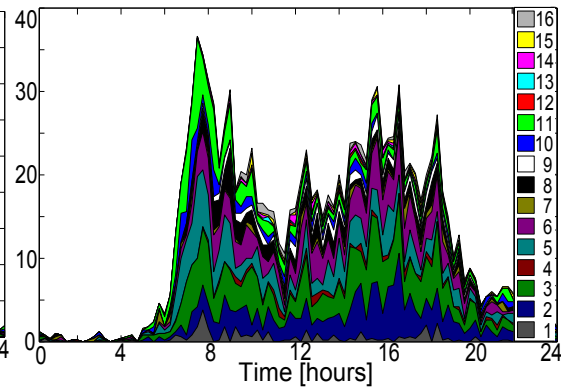

(c) Contribution of each road to the total queue length for the self-organized policy.

Figure 2: Comparison of total queue lengths and road contributions to total queue lengths for both policies in simulation.

assumed to follow a nonhomogeneous Poisson process. The network consists of three intersections, which all are regulated by traffic lights. The roads (or lanes) are labeled from 1 up to 17 , however road 17 has no traffic light and will not be shown in the results. The network is divided over three intersections, $\mathrm{a}, \mathrm{b}$, and $\mathrm{c}$.

\subsection{RESULTS}

All figures are the result of the average of ten runs of one day. Figure 2a shows the total queue length of all roads for the two different policies with a constant service rate of 0.45 cars per second. From this, we see the simulated ex-

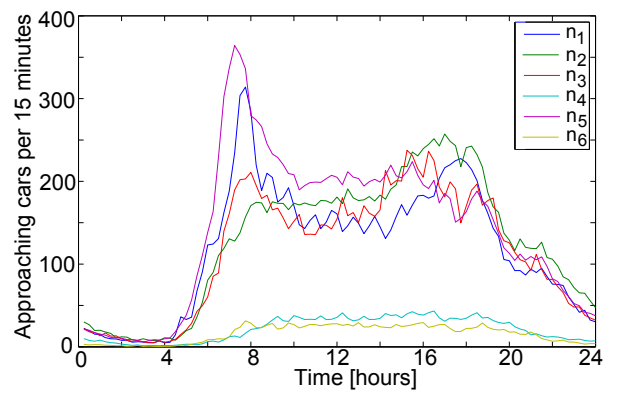

Figure 3: Estimated average daily arrival of cars at intersection a.

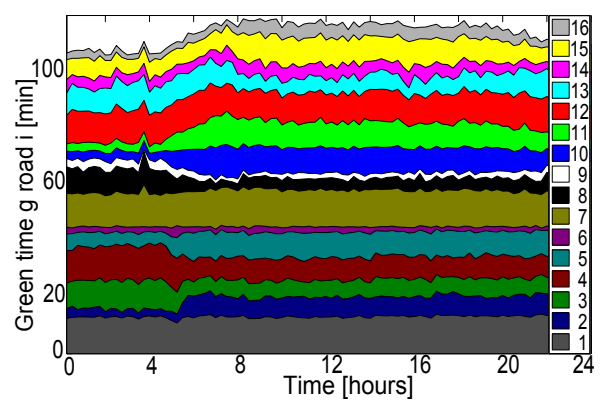

Figure 4: Simulated green time of each road for the selforganized policy.

pected total number of cars in queue in the morning is much higher for the SCATS-like policy than for the self-organized policy. However, the actual difference would probably be smaller, because we assumed that the SCATS-like policy uses fixed green times, which is less flexible than the real
SCATS. To examine the differences between the two policies the total queue length of each road for each policy will be compared. Figures $2 \mathrm{~b}$ and $2 \mathrm{c}$ show the contribution of each road to the total queue length under the two policies; note that the figures have different scales. The figures show that the SCATS-like policy has a large queue on road 11; this is not observed for Lämmer's policy in which the queue length is more balanced between the different roads.

To get a better understanding of the behavior of Lämmer's policy the minutes of green time for each road during 15 minutes is studied; see Figure 4 . This figure shows the adjustments of Lämmer's policy to the arrival rate of traffic at intersection a over the course of a typical week day in September 2014. As is shown in Figure 3, the number of approaching cars to intersection a is increasing around $4 \mathrm{am}$, which also shows up in the self-organized policy at this time as a new apportioning of the green times in Figure 4.

\section{OUTLOOK}

From this preliminary study it can be concluded that Lämmer's policy improves traffic flows and is promising for the future of adaptive traffic control in congested road networks. For further research it would be interesting to investigate what effect unpredictable (adaptive) traffic lights have on drivers; it may cause irritation or confusion when one lane is served twice before another lane is served.

\section{ACKNOWLEDGEMENTS}

We thank Yoni Nazarathy for initiating this Dutch-Australian collaboration through partial support of Australian Research Council (ARC) Grants DP130100156 and DE130100291.

\section{REFERENCES}

[1] J. de Gier, T. M. Garoni, and O. Rojas. Traffic flow on realistic road networks with adaptive traffic lights. Journal of Statistical Mechanics: Theory and Experiment, 2011(04):P04008, 2011.

[2] S. Lämmer and D. Helbing. Self-control of traffic lights and vehicle flows in urban road networks. Journal of Statistical Mechanics: Theory and Experiment, 2008(04):P04019, 2008.

[3] A. G. Sims and K. Dobinson. The Sydney coordinated adaptive traffic (SCAT) system philosophy and benefits. Vehicular Technology, IEEE Transactions on, 29(2):130-137, 1980. 